\title{
Heterochronic Spontaneous Rupture of Bilateral Renal Cell Carcinomas in a Hemodialysis Patient
}

\author{
Shunsuke Yamada ${ }^{1,2}$, Hideki Yotsueda ${ }^{3}$, Tadashi Nagara ${ }^{3}$, Ken Goto $^{4}$, Yutaka Nakashima ${ }^{5}$, \\ Hideki Hirakata ${ }^{3}$, Satoru Fujimi ${ }^{1}$ and Kazuhiko Tsuruya ${ }^{6}$
}

\begin{abstract}
A 64-year-old man undergoing chronic hemodialysis was admitted under a shock state with macrohematuria and fatigue lasting for two hours. A blood analysis revealed severe anemia. Computed tomography disclosed a large right-sided perirenal hematoma. The patient was successfully treated with radical nephrectomy, leading to a histological diagnosis of spontaneous rupture of renal cell carcinoma (RCC). One year after rupture of the right RCC, he again developed macrohematuria and computed tomography revealed a left-sided perirenal hematoma. Radical nephrectomy followed by a histological examination revealed spontaneous rupture of the left-sided RCC. This case emphasizes the importance of conducting periodic imaging evaluations of chronic hemodialysis patients with renal cystic masses.
\end{abstract}

Key words: acquired cystic disease of the kidney, end-stage renal disease, hemodialysis, hemorrhagic shock, renal cell carcinoma

(Intern Med 52: 667-671, 2013)

(DOI: 10.2169/internalmedicine.52.8703)

\section{Introduction}

Renal cell carcinoma (RCC) is an occasional lifethreatening complication in patients undergoing dialysis and is one of the most common neoplasms in Japanese hemodialysis patients (1). RCCs occurring in hemodialysis patients are often accompanied by acquired cystic disease of the kidney (ACDK) (2), which increases in prevalence with increasing duration of dialysis and appears to be an origin of RCC (3). Patients with RCC typically present with symptoms such as macroscopic hematuria, flank pain and fever; however, few patients present with all three symptoms.

Recent advances in imaging technology allow for the detection of RCCs before patients become symptomatic, and increasing numbers of RCCs are now detected fortuitously in the general population on computed tomography (CT) or ultrasonography during the course of other investigations (4). However, RCCs are often difficult to distinguish from complicated, infected cysts or hemorrhagic cysts in hemodialysis patients, even using dynamic CT and/or magnetic resonance imaging (MRI) (5). Hence, even in this era of advanced imaging, some patients occasionally show atypical presentations, including hemorrhagic shock related to spontaneous rupture of RCC during the follow-up pe$\operatorname{riod}(6)$.

We herein report a rare case of a hemodialysis patient with bilateral RCCs fortuitously detected on CT who developed two distinct episodes of hemorrhagic shock related to spontaneous and metachronous rupture of the RCCs in both kidneys.

\section{Case Report}

A 64-year-old man undergoing chronic hemodialysis was admitted under a shock state to our emergency room due to fatigue, macrohematuria and right flank pain lasting for the previous two hours. He had been receiving hemodialysis for

\footnotetext{
${ }^{1}$ Fukuoka Renal Clinic, Japan, ${ }^{2}$ Division of Internal Medicine, Fukuoka Dental College, Japan, ${ }^{3}$ Division of Nephrology, Japanese Red Cross Fukuoka Hospital, Japan, ${ }^{4}$ Division of Urology, Japanese Red Cross Fukuoka Hospital, Japan, ${ }^{5}$ Division of Pathology, Japanese Red Cross Fukuoka Hospital, Japan and ${ }^{6}$ Department of Integrated Therapy for Chronic Kidney Disease, Graduate School of Medical Sciences, Kyushu University, Japan

Received for publication July 29, 2012; Accepted for publication November 16, 2012

Correspondence to Dr. Kazuhiko Tsuruya, tsuruya@intmed2.med.kyushu-u.ac.jp
} 
Table 1. Laboratory Data Obtained on the First Admission

\begin{tabular}{|c|c|c|c|}
\hline Complete blood count & & Creatinine (mg/dL) & 11.8 \\
\hline Hemoglobin $(\mathrm{g} / \mathrm{dL})$ & 6.6 & Uric acid (mg/dL) & 7.2 \\
\hline Hematocrit (\%) & 19.5 & Sodium (mmol/L) & 138 \\
\hline White blood cell $(/ \mu \mathrm{L})$ & 8,700 & Potassium (mmol/L) & 5.3 \\
\hline Platelet $(/ \mu \mathrm{L})$ & 67,000 & Chloride (mmol/L) & 107 \\
\hline Biochemistry & & Calcium (mg/dL) & 7.6 \\
\hline Total protein $(\mathrm{g} / \mathrm{dL})$ & 4.7 & Phosphorus (mg/dL) & 8.3 \\
\hline $\operatorname{Albumin}(\mathrm{g} / \mathrm{dL})$ & 2.3 & Creatinine kinase (U/L) & 14 \\
\hline Total bilirubin (mg/dL) & 0.2 & Amylase (U/L) & 100 \\
\hline Direct bilirubin (mg/dL) & 0.1 & Glucose (mg/dL) & 127 \\
\hline Aspartate aminotransferase (U/L) & 13 & C-reactive protein (mg/dL) & 0.4 \\
\hline Alanine aminotransferase (U/L) & 14 & Blood gas analysis & \\
\hline Lactate dehydrogenase (U/L) & 139 & $\mathrm{pH}$ & 7.31 \\
\hline Alkaline phosphatase (U/L) & 195 & $\mathrm{pO}_{2}(\mathrm{mmHg})$ under $5 \mathrm{~L} / \mathrm{min}$ oxygen & 300 \\
\hline Blood urea nitrogen $(\mathrm{mg} / \mathrm{dL})$ & 65.7 & $\mathrm{pCO}_{2}(\mathrm{mmHg})$ & 27.8 \\
\hline
\end{tabular}

end-stage renal disease caused by chronic glomerulonephritis three times a week for the last 12 years. His past medical history included percutaneous transcatheter coronary intervention for ischemic heart disease three months prior to admission. His blood pressure level during hemodialysis fluctuated between 120-130/70-80 mmHg. His medication regimen included aspirin for secondary prevention of ischemic heart disease. Two months prior to presentation, a right renal mass suspicious of RCC had been fortuitously detected on enhanced CT. However, at that time, the renal mass was not distinguished from the complicated cysts often seen in hemodialysis patients with ACDK and was therefore left untreated.

On presentation, the patient appeared pale but alert. His blood pressure was $84 / 53 \mathrm{mmHg}$, his heart rate was 84 beats per minute and his body temperature was $36.9^{\circ} \mathrm{C}$. His height was $163.2 \mathrm{~cm}$ and his body weight was $47.4 \mathrm{~kg}$. The palpebral conjunctiva was anemic. The results of laboratory tests performed on admission are listed in Table 1. Most of the data were compatible with hemodialysis, except for the extremely low hemoglobin level $(6.6 \mathrm{~g} / \mathrm{dL})$. Enhanced CT disclosed a large right-sided perirenal mass, suggesting the presence of a perirenal hematoma caused by rupture of the right renal mass (Fig. 1). Because the patient remained in a state of hemorrhagic shock, we performed right nephrectomy with a blood transfusion, even though imaging could not confirm a diagnosis of RCC rupture. The patient recovered from hemorrhagic shock following the procedure. The right kidney is shown in Fig. 2A. A macroscopic examination showed a poorly-demarcated tumor measuring $2.8 \mathrm{~cm}$ at the maximum diameter. A histological examination revealed atypical cells with clear or faintly eosinophilic cytoplasm proliferating in a sinusoidal or tubular pattern, leading to a final diagnosis of RCC (clear cell carcinoma) (Fig. 3A, B). The staging was pT1a, G2, INF $\beta, \mathrm{v}(-)$. Aspirin had been discontinued during hospitalization and was re-started at the time of discharge. However, enhanced CT performed after right nephrectomy disclosed several cysts with solid-like portions located in the upper part of the left kidney. Because enhanced CT could not differentiate between RCC and com- plicated cysts, a decision to perform preemptive left nephrectomy was considered. However, the patient was scheduled to receive periodic CT evaluations after discharge, and left nephrectomy was therefore postponed due to the risk of intractable persistent hypotension following bilateral nephrectomy.

Six months after rupture of the right RCC, enhanced CT showed enlargement of the complicated cysts in the left kidney over a 6-month period, suggesting the presence of an RCC in the left kidney, and elective left nephrectomy was therefore scheduled. However, the patient was admitted to our hospital for low back pain and macrohematuria eight days after the last CT evaluation. The hemoglobin level at the second admission was $10.6 \mathrm{~g} / \mathrm{dL}$. Enhanced CT revealed a left perirenal hematoma, suggesting rupture of the known left cystic mass. The patient was again treated successfully with left radical nephrectomy and a blood transfusion. The left kidney is shown in Fig. 2B. A histological diagnosis of RCC (clear cell carcinoma) was finally confirmed (Fig. 3C, D). The stage was pT2, G2, INF $\beta$, v(-). The patient was discharged after 10 days of hospitalization and has continued to receive hemodialysis therapy at our clinic three times a week. He has remained in good health without relapse of RCC for four years.

\section{Discussion}

This report presents the rare case of a hemodialysis patient with bilateral RCCs fortuitously detected on CT who subsequently presented with two distinct episodes of hemorrhagic shock related to metachronous and spontaneous rupture of the bilateral RCCs. Each episode of rupture was successfully treated with radical nephrectomy and an adequate blood transfusion. The current case highlights the difficulty of making a correct preoperative differentiation between RCCs and complicated cysts and alerts clinicians to the potential clinical features of RCC, including spontaneous rupture.

Various kidney diseases can rupture spontaneously, leading to the development of perirenal or subcapsular hema- 


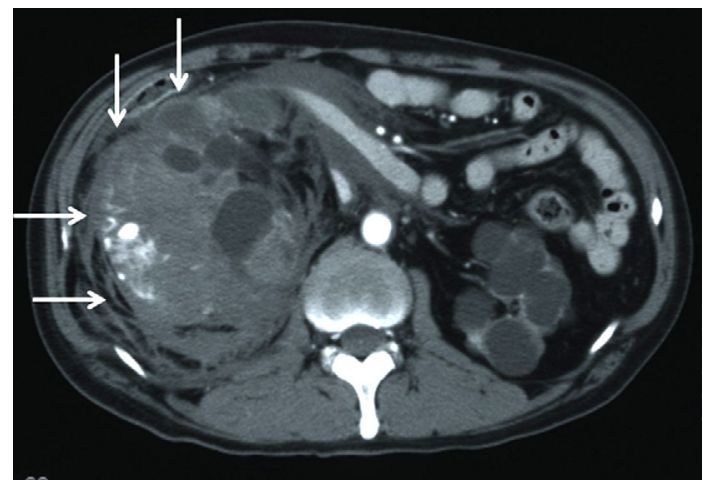

Figure 1. Computed tomography of the abdomen performed on the first admission showing ruptured RCCs . The white arrows show the hematoma around the right kidney. The white intensity around the kidney indicates leakage of contrast medium.

tomas or hemorrhage. The most common types of rupture responsible for perirenal hemorrhage in the general population are ruptured angiomyolipomas (33\%), closely followed by RCCs (26\%) and vascular diseases (26\%) (7). However, data for dialysis patients are scarce, although angiomyolipomas, RCCs and renal cysts have been reported as causes of perirenal or subcapsular hemorrhages (8). Although the precise mechanisms underlying renal mass rupture remain undetermined, dialysis patients seem to be particularly prone to this complication due to the administration of heparin during dialysis and because their platelet function is impaired. Furthermore, uncontrolled hypertension, which is frequently observed in dialysis patients, can accelerate the development of RCC rupture. In the present case, the patient's blood pressure before the RCC ruptures was well-controlled. However, aspirin use for the secondary prevention of ischemic heart disease and intermittent heparin use during hemodialysis sessions may have increased the risk of rupture.

Spontaneous RCC rupture occasionally occurs in dialysis patients (5), and nine cases have been reported to date, although seven of these are only available in the Japanese literature (Table 2). All 10 cases, including the current case, involved men, with dialysis durations of more than nine years. All cases were associated with ACDK. The size of the ruptured RCC ranged from a small mass $<1 \mathrm{~cm}$ in diameter to a large mass $>5.5 \mathrm{~cm}$ in diameter. Regarding the mechanisms of RCC rupture, several theories have been proposed, including congestion of the renal veins due to obstruction by the RCC, vascular extension and tearing by rapid enlargement of the RCC and direct invasion of the RCC into the renal vasculature. In the present case, there were no histological findings of vascular invasion or other uncommon histological features. The gross appearance of the bilateral kidneys involved atrophy with multiple cysts. The age and sex of the patient and the duration of dialysis in the present case were similar to those reported in cases of unruptured RCCs. However, because the clear-cell type is often hypervascular and prone to decay, the histological type of RCC may have
A

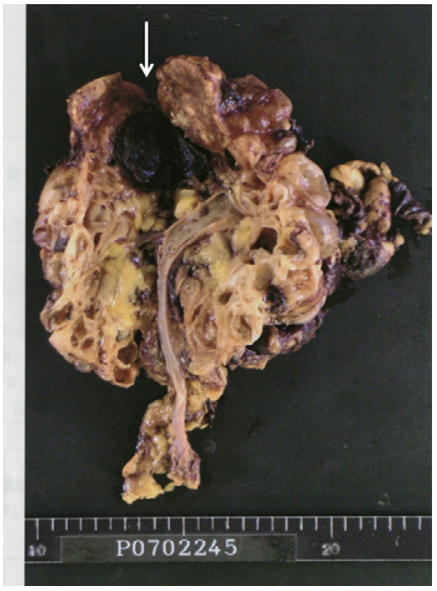

B

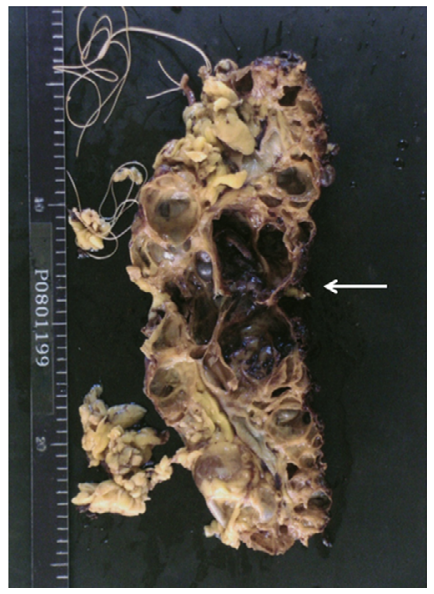

Figure 2. Macroscopic photographs of the surgically resected right (A) and left (B) kidneys fixed with formalin. The arrows indicate the ruptured RCCs in each kidney.

increased the risk of rupture; most ruptured cases, including the present case and the cases noted in the literature review, were the clear-cell type.

The RCC in the present case was believed to have originated from ACDK and developed bilaterally. One of the important dilemmas associated with this case is whether the second rupture of the RCC in the left kidney was avoidable. Approximately $80 \%$ of RCCs in hemodialysis patients are related to ACDK. ACDK-related RCCs are prone to be multi-centric (9), and approximately $10 \%$ of patients who develop RCCs based on ACDK subsequently develop RCCs in the contralateral side (10). Some urologists propose the use of preventive surgery to treat RCCs; however, others prefer to avoid preventive surgery because RCC resection is invasive and bilateral nephrectomy occasionally leads to uncontrollable persistent hypotension after surgery.

The problem of differentiating between RCC and other types of renal cystic masses, including complicated cysts, in dialysis patients using CT or ultrasonography remains an important clinical issue. There are currently no biomarkers that can differentiate RCC from other neoplastic or cystic diseases in the kidneys, and imaging techniques remain the most important source of information in the course of preoperative diagnosis (11). MRI and dynamic-contrast CT have greatly improved the rates of correct and early diagnoses of RCC. However, a decisive diagnosis of RCC independent of histological confirmation is relatively hard to make: RCC rupture occurred twice in the present case before the diagnosis of RCC was confirmed with imaging. Therefore, developing further decisive imaging techniques and biomarkers is required. The repeated use of combined imaging technologies therefore currently represents the most realistic and reasonable approach (12).

Another important issue in the present case was the origin of the bilateral RCCs. We should consider two possibilities: the bilateral RCCs may have developed independently or one RCC may have arisen through metastasis from the ipsi- 
A

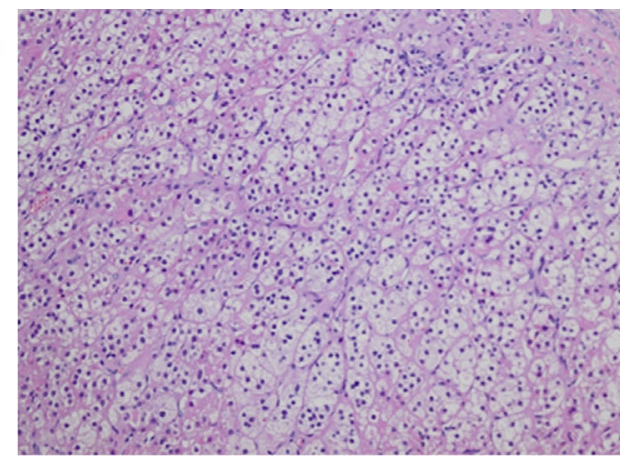

C

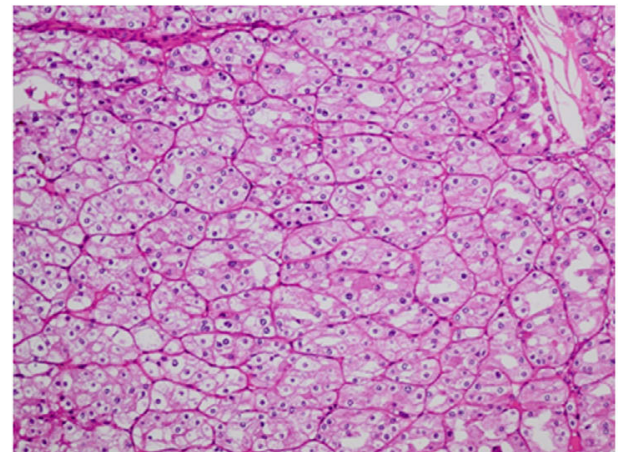

$\mathrm{B}$

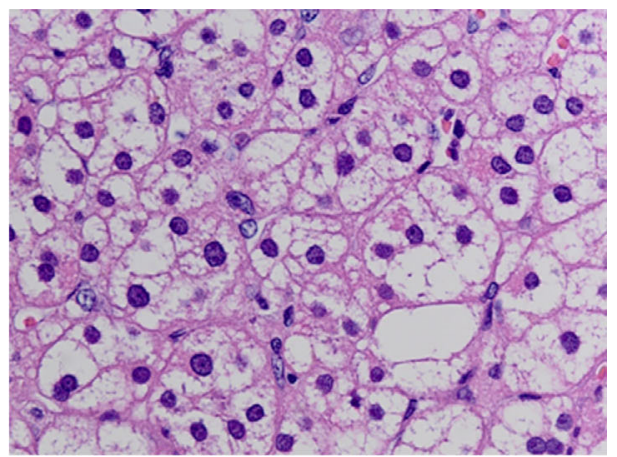

$\mathrm{D}$

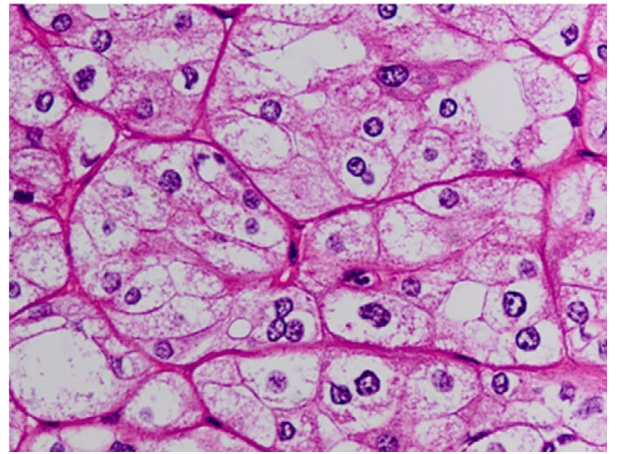

Figure 3. Photomicrographs of each RCC (Hematoxylin-eosin staining). Both the right (A, B) and left (C, D) RCCs were histologically diagnosed as clear-cell RCCs. Original magnifications: ×100 (A, C) and $\times 400(B, D)$.

Table 2. Literature Review of Cases of RCC Rupture in Hemodialysis Patients

\begin{tabular}{|c|c|c|c|c|c|c|c|c|c|}
\hline Patient & Author & $\begin{array}{l}\text { Publication } \\
\text { (year) }\end{array}$ & $\begin{array}{l}\text { Age } \\
\text { (years) }\end{array}$ & Sex & $\begin{array}{l}\text { Duration } \\
\text { (years) }\end{array}$ & Side & Treatment & Histology & Stage \\
\hline 1 & Eto $\mathrm{H}$ & 1986 & 61 & male & 10 & right & $\mathrm{Nx}$ & clear cell & No data \\
\hline 2 & Shiba M & 1997 & 41 & male & 16 & left & $\mathrm{Nx}$ & cystic & pT2pN0M0 \\
\hline 3 & Yonou K & 1999 & 53 & male & No data & left & $\mathrm{Nx}$ & papillary & No data \\
\hline 4 & Nishikai S & 2000 & 65 & male & No data & right & TAE, Nx & clear cell & No data \\
\hline 5 & Matsui F & 2003 & 67 & male & 13 & right & $\mathrm{Nx}$ & papillary & No data \\
\hline 6 & Goto $\mathrm{T}$ & 2009 & 50 & male & 12 & right & $\mathrm{Nx}$ & No data & No data \\
\hline 7 & Otsuki H & 2011 & 55 & male & 9 & left & $\mathrm{Nx}$ & No data & No data \\
\hline 8 & Kim WB & 2011 & 47 & male & 15 & left & $\mathrm{Nx}$ & clear cell & pT1apN0M0 \\
\hline 9 & Kim WB & 2011 & 49 & male & 10 & left & TAE, Nx & clear cell & pT1apN0M0 \\
\hline \multirow[t]{2}{*}{ Current } & Yamada S & 2012 & 64 & male & 12 & right & $\mathrm{Nx}$ & clear cell & pT1apN0M0 \\
\hline & & & & & & left & $\mathrm{Nx}$ & clear cell & pT2pN0M0 \\
\hline
\end{tabular}

lateral side. However, it is difficult to distinguish between these options in the present case. Unfortunately, both bilateral RCCs were histologically clear-cell type and of the same cytological grade. Furthermore, even if one RCC arose by metastasis from the ipsilateral RCC, it would not be possible to determine which was the primary site because the left RCC was detected at the time of the right RCC rupture.

The treatment of ruptured RCCs has been changing in line with advances in interventional radiology. Recent clinical experience has shown that percutaneous angiography followed by transcatheter arterial embolization (TAE) can successfully manage bleeding, leading to elective radical nephrectomy (13). These authors proposed that the use of preoperative TAE should be considered in the event of hem- orrhagic shock because it allows for control of the hemorrhage and a clean surgical field and reduces the amount of intraoperative blood loss. Furthermore, preoperative TAE allows for a clear view of the affected lesion after hemostasis, thus increasing the number of opportunities to confirm the diagnosis. Fortunately, the present patient was able to undergo radical nephrectomy with an adequate blood transfusion, despite being in hemorrhagic shock on admission. However, in cases of retroperitoneal or perirenal hemorrhage with unstable hemodynamics, preoperative TAE can be considered to be one of the therapeutic options.

We herein reported the rare case of a patient under chronic hemodialysis who developed two distinct episodes of RCC rupture. Physicians should be aware of the possibil- 
ity of spontaneous rupture of RCCs in dialysis patients, and patients with ACDK with the potential to develop into RCC should be carefully monitored. The use of repeated evaluations with enhanced CT is recommended if complicated cysts or other benign masses cannot be easily distinguished from RCC in dialysis patients.

The authors state that they have no Conflict of Interest (COI).

\section{Acknowledgement}

We would like to thank the Edanz Group (http://www. edanzediting.co.jp/) for their careful reading and editing of this manuscript.

\section{References}

1. Satoh S, Tsuchiya N, Habuchi T, et al. Renal cell and transitional cell carcinoma in a Japanese population undergoing maintenance dialysis. J Urol 174: 1749-1753, 2005.

2. Levine E, Slusher SL, Grantham JJ, Wetzel LH. Natural history of acquired renal cystic disease in dialysis patients: a prospective longitudinal CT study. AJR 156: 501-506, 1991.

3. Dunnill MS, Millard PR, Oliver D. Acquired cystic disease of the kidneys: A hazard of long-term intermittent maintenance haemodialysis. J Clin Pathol 30: 868-877, 1997.

4. Heidenreich A, Ravery V. Preoperative imaging in renal cell can- cer. World J Urol 22: 307-315, 2004.

5. Israel GM, Hindman N, Bosniak MA. Evaluation of cystic renal masses: comparison of $\mathrm{CT}$ and MR imaging by using the Bosniak classification system. Radiology 231: 365-371, 2004.

6. Kim WB, Lee ES, Doo W, et al. Spontaneuously ruptured renal cell carcinoma during hemodialysis in two patients with end-stage renal disease. Korean J Urol 52: 865-867, 2011.

7. Yip KH, Peh WC, Tam PC. Spontaneous rupture of renal tumors: the role of imaging in diagnosis and management. Br J Radiol 71: 146-154, 1998.

8. Levine E, Grantham JJ, MacDougall ML. Spontaneous subcapsular and perinephric hemorrhage in end-stage kidney disease: clinical and CT findings. AJR 148: 755-758, 1987.

9. Ikeda R, Tanaka T, Moriyama MT, et al. Proliferative activity of renal cell carcinoma associated with acquired cystic disease of the kidney: comparison with typical renal cell carcinoma. Human Pathol 33: 230-235, 2002.

10. Truong LD, Bhuvaneswari K, Cao JTH, Barrios R, Suki WN. Renal neoplasma in acquired cystic kidney disease. Am J Kidney Dis 26: 1-12, 1995.

11. Levine E, Slusher SL, Grantham JJ, Wetzel LH. Natural history of acquired renal cystic disease in dialysis patients: a prospective longitudinal CT study. AJR 156: 501-506, 1991.

12. Yip KH, Peh WCG, Tam PC. Spontaneous rupture of renal tumors: the role of imaging in diagnosis and management. British $\mathrm{J}$ Radiol 71: 146-154, 1998.

13. Habib M. Arterial embolization for spontaneous rupture of renal cell carcinoma. Saudi J Kidney Dis Transpl 22: 1243-1245, 2011.

(C) 2013 The Japanese Society of Internal Medicine http://www.naika.or.jp/imonline/index.html 